University of Wollongong

Research Online

Faculty of Engineering and Information

Faculty of Engineering and Information

Sciences - Papers: Part B

Sciences

2020

Design and experimental evaluation of a new modular underactuated multifingered robot hand

Shufeng Tang

Yue Yu

Shuaishuai Sun

Zhixiong Li

University of Wollongong, lizhixio@uow.edu.au

Thompson Sarkodie-Gyan

See next page for additional authors

Follow this and additional works at: https://ro.uow.edu.au/eispapers1

Part of the Engineering Commons, and the Science and Technology Studies Commons

Research Online is the open access institutional repository for the University of Wollongong. For further information contact the UOW Library: research-pubs@uow.edu.au 


\title{
Design and experimental evaluation of a new modular underactuated multi- fingered robot hand
}

\author{
Abstract \\ (C) IMechE 2020. In this paper, a modular underactuated multi-fingered robot hand is proposed. The robot \\ hand can be freely configured with different number and configuration of modular fingers according to the \\ work needs. Driving motion is achieved by the rigid structure of the screw and the connecting rod. A \\ finger-connecting mechanism is designed on the palm of the robot hand to meet the needs of modular \\ finger's installation, drive, rotation, and sensor connections. The fingertips are made of hollow rubber to \\ enhance the stability of grasping. Details about the design of the robot hand and analysis of the robot \\ kinematics and grasping process are described. Last, a prototype is developed, and a grab test is carried \\ out. Experimental results demonstrate that the structure of proposed modular robot hand is reasonable, \\ which enables the adaptability and flexibility of the modular robot hand to meet the requirements of \\ various grasping modes in practice.

\section{Disciplines} \\ Engineering | Science and Technology Studies

\section{Publication Details} \\ Tang, S., Yu, Y., Sun, S., Li, Z., Sarkodie-Gyan, T. \& Li, W. (2020). Design and experimental evaluation of a \\ new modular underactuated multi-fingered robot hand. Proceedings of the Institution of Mechanical \\ Engineers, Part C: Journal of Mechanical Engineering Science,
}

\section{Authors}

Shufeng Tang, Yue Yu, Shuaishuai Sun, Zhixiong Li, Thompson Sarkodie-Gyan, and Weihua Li 

Multi-fingered Robot Hand

3 Shufeng Tang ${ }^{1}$, Yue $\mathrm{Yu}^{1}$, Shuaishuai Sun ${ }^{2}$, Zhixiong $\mathrm{Li}^{3}{ }^{3}{ }^{*}$, Thompson Sarkodie-Gyan ${ }^{3}$, Weihua $\mathrm{Li}^{3}$

4 1. School of Mechanical Engineering, Inner Mongolia University of Technology, Hohhot 010051,

\section{China}

6 2. New Industry Creation Hatchery Center, Tohoku University, Sendai 980-8577, Japan

7 3. School of Mechanical, Materials, Mechatronic and Biomedical Engineering, University of 8 Wollongong, NSW 2500, Australia

9 4. College of Engineering, University of Texas, $500 \mathrm{~W}$ University Ave, El Paso, TX 79968, USA

10 *Correspondence: zhixiong_li@uow.edu.au

Abstract: In this paper, a modular underactuated multi-fingered robot hand is proposed. The robot hand can be freely configured with different number and configuration of modular fingers according to the work needs. Driving motion is achieved by the rigid structure of the screw and the connecting rod. A Finger connecting mechanism is designed on the palm of the robot hand to meet the needs of modular finger's installation, drive, rotation, and sensor connections. The fingertips are made of hollow rubber to enhance the stability of grasping. Details about the design of the robot hand and analysis of the robot kinematics and grasping process are described. Lastly, a prototype is developed and a grab test is carried out. Experimental results demonstrate that the structure of proposed modular robot hand is reasonable, which enables the adaptability and flexibility of the modular robot hand to meet the requirements of various grasping modes in practice.

Keywords: underactuated; multi-fingered; robot hand; kinematic analysis; grab simulation;

\section{Introduction}

With the development of robotics, providing robots with a pair of smart hands has gradually become a research topic of great interest. Multi-finger dexterous hands have more contact points with objects. If appropriate gripping methods and algorithms are adopted, theoretically, the grasping and manipulation of objects of any shape can be completed. Therefore, the multi-finger robot hand can perform the grasping and manipulation of various complicated-shape objects with high speed, stability, and reliability without replacing the end effector.

At present, robot hands can be divided into two kinds according to their driving modes: fully actuated robot hand and underactuated robot hand. Among them, the fully actuated robot hand has independent driving sources at each finger joint, and each joint can be independently controlled. The advantage of full-drive is that the robot hand has higher controllability. In particular, when the robot hand grasps an object, it can control the gripping process more precisely by controlling the speed and movement angle of the joint, so that the robot hand can grasp the target almost perfectly. There are some typical fully actuated robot hands: such as Gifu-III [1,2] from Japan's Gifu University, the Dexhand robot hand [3] developed by German Aerospace Center, Robonaut 2 [4, 5] developed by NASA, China HIT/DLR-II [6, 7] developed by Harbin Institute of Technology, and Sandia Hand [8] 
An underactuated robot hand means that the number of driving sources is smaller than the DOFs

(degrees of freedoms) of robot hand. The finger relies on the underactuated finger mechanism to achieve passive adaptation to the shape of the grasped object during grasping. It requires only a small number of drive components and a simple control system to have a wide range of gripping and good load capacity. Examples of such a system include the shadow robot hand [9] developed by Shadow Company, the MPJ robot hand [10], the GUCA robot [11], the PCSS [12] and PASA robots [13] developed by Tsinghua University, the prosthetic hand [14] developed by Bogazici University, IHYHAND [15] developed by Yale University, uGRIPP [16] developed by Tohoku University, the Pisa/IIT SoftHand 2 [17] developed by University of Pisa, and the ISR-SoftHand [18] developed by University of Coimbra.

In recent years, robot hands using soft materials have attracted widespread attentions worldwide [19-21]. The soft hand fully exerts the natural flexibility of various flexible materials in the manufacturing process and fully utilizes the nonlinearity, viscoelasticity, and hysteresis of the material in the movement and control of the soft robot hand, which simplifies the complexity of the robot hand control system and increases the flexibility and interactivity of the robot hands. Some typical applications include the RBO hand 2 [22] of the University of Berlin, the OS-HAND fourfinger software hand [23] and the CSTA-II software robot hand [24] of Tsinghua University, BCL13 [25] developed by University of Hong Kong, and a software robot [26] developed by the University of Colorado.

Most of the existing robot hands only have one set of working mode [27-32]. If the working mode changes, one has to replace the robot hand. The replacement process is very cumbersome, and the control system of the traditional robot hand is complicated because in order to determine the gripping posture many sensors need to acquire force, position, torque information and so forth [3338]. As a result, applicable robot hands that can be adapted to variable configurations and working modes remain a challenge.

Different from the full flexible robot hands, the motor-driven robot hand does not need air source. So it is more convenient to install and use and can provide greater grasping force. Compared with the full rigid robot hands, the rigid flexible coupling robot hand has better safety in human-computer interaction and can operate on fragile objects. This paper presents a modular underactuated multi fingered robot hand, which can be configured with different numbers and structures of fingers according to work requirements. The mechanical finger adopts the rigid flexible coupling structure design, which further improves the grasping ability and safety of the robot hand. The finger rotating mechanism is designed to widen the operation range of the robot hand. Because the motor-driven robot hand needs to meet the needs of finger fixation, driving, rotation and sensor connection when changing fingers, the present robot hand is more complex in modularity. The installation mechanism of the robot hand is designed on the palm to meet the requirements of modular finger installation, drive, rotation and sensor connection. Using denavit-hartenberg (D-H) model, the fingertip trajectory is deduced and the workspace of manipulator is analyzed. The grasping process of the robot hand is analyzed theoretically and simulated. The rationality of the modular robot hand structure is evaluated by studying the swing angle and contact force of the joint in the grasping process. Experiments show that the manipulator can grasp different shapes of objects conveniently and safely. 
Modular underactuated multi-finger robot hand is robotic hand designed with a modular concept. The modular underactuated robot hand consists of modular fingers and palm, and the finger's rotation mechanism is additionally designed on the palm to improve the adaptability of the robot hand to a long object. The modular underactuated robot hand adopts an underactuated driving method, and the palm design has six finger-mounting ports, so that modular fingers of different numbers and configurations can be freely configured according to the work requirements. Different hand configurations are shown in Figure 1.

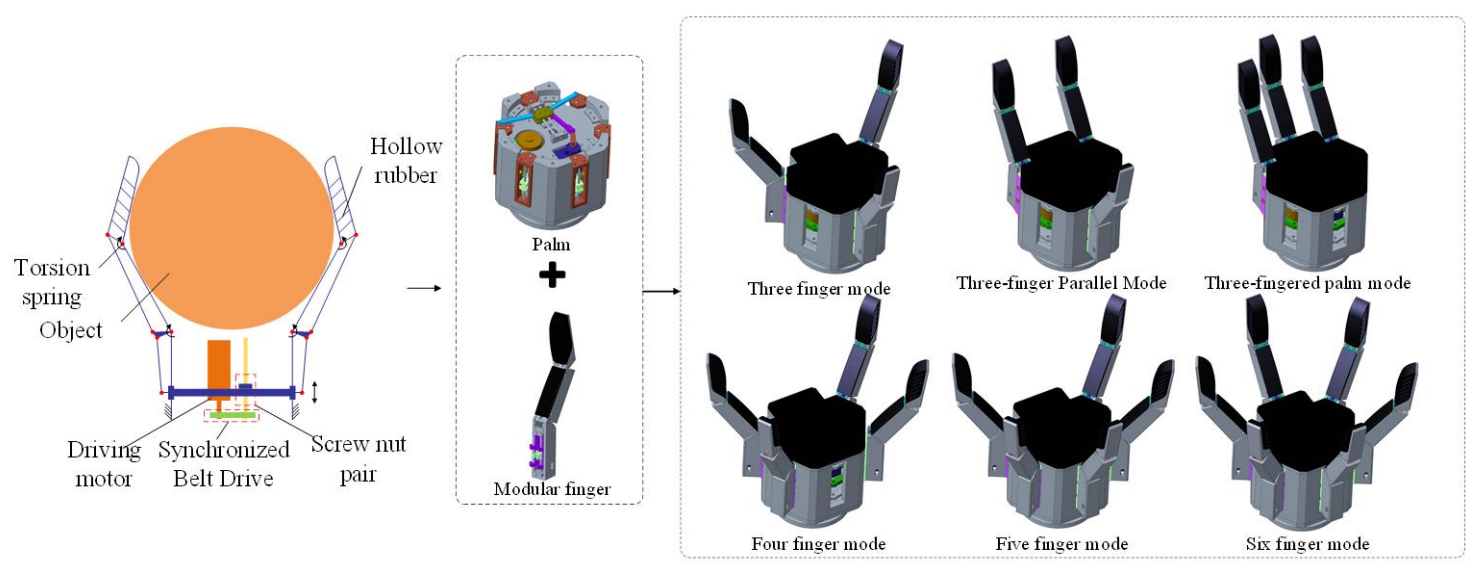

Figure 1. The structure figure of the robot hand configuration.

\subsection{The design of the drive system}

The robot hand adopts underactuated driving mode, in which a single motor is required to drag multiple fingers. The motor is connected to the lead screw nut through the timing belt drive, and the nut on the lead screw is connected with the intermediate sliding plate to change the rotary motion of the motor into the up and down movement of the sliding plate, and the middle sliding plate pushes the driving slider of the modular finger up and down and control the finger to open and close. The drive structure of the robot hand is shown in Figure 2(a).

The different gripping modes of the robot hand are achieved by the finger rotating mechanism. After the finger is rotated, the robot hand's grasp changes from the original centering grasp to a parallel grasp, which enhances the adaptability of the robot hand to long-shaped objects and expands the scope of robot hand. The schematic diagram of the finger rotating mechanism is shown in Figure 2(b).

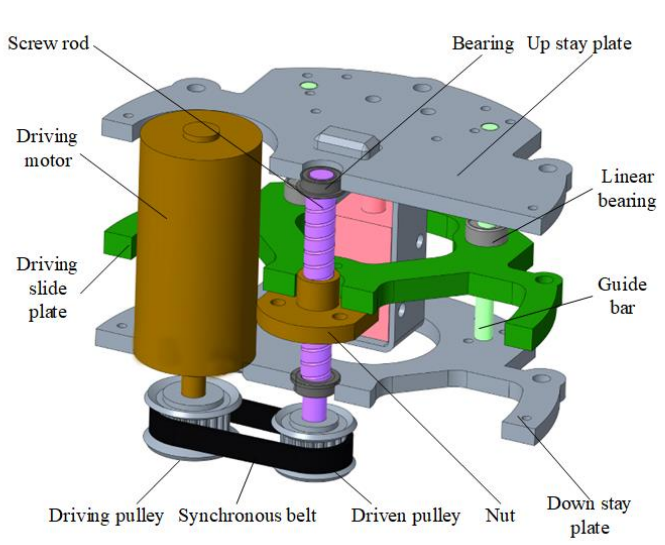

(a) The drive structure of the robot hand

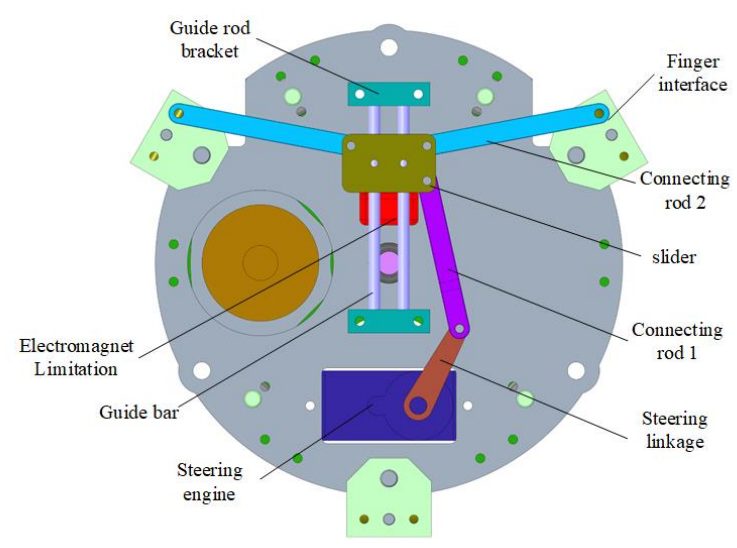

(b) The finger rotating mechanism 


\subsection{The design of the modular finger}

The modular finger adopts a rigid-flexible multi-rod hybrid series connection, the fingertip is replaced by a flexible hollow rubber structure. During the grasping process, the robot hand are brought into contact with the two surfaces by the original two points, which increases the contact area between the finger and enhances the stability of the grasping process. The modular fingers of the robot hand are shown in Figure 3(a).

The modular underactuated robot hand can be freely configured with different numbers and structure of modular fingers depending on the job requirements. An additional finger interface is designed on the palm of the robot hand to meet the needs of modular finger's installation, drive, rotation, and sensor connections. The finger interface is shown in Figure 3(b).

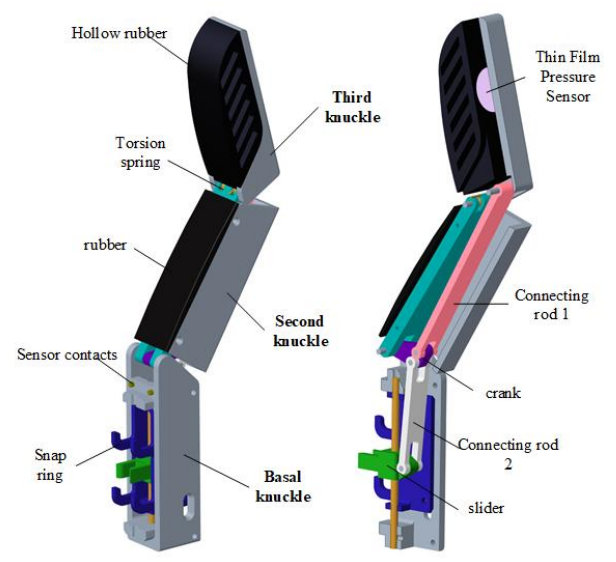

(a) The modular fingers
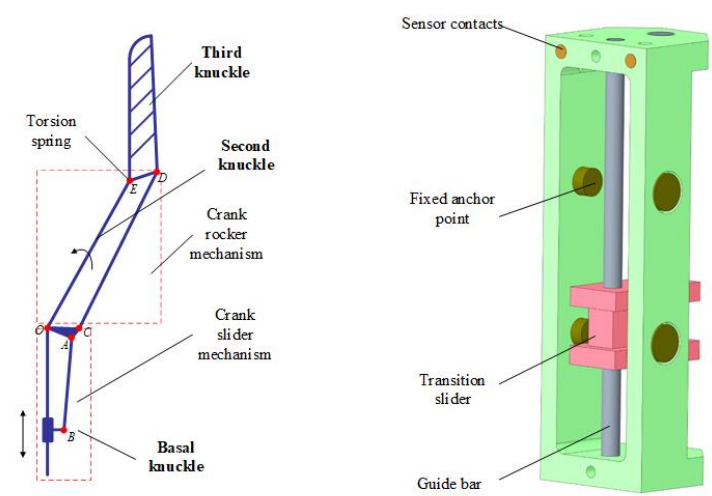

(b) The finger interface

Figure 3. Structure diagram of the modular finger.

The finger installation process is shown in Figure 4.

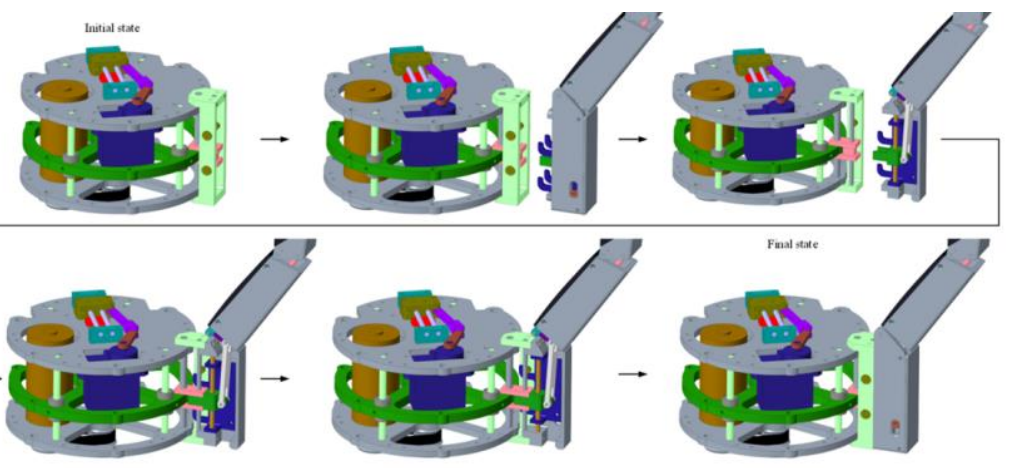

Figure 4. Installation process of modular finger.

\section{Motion and mechanics analysis of the modular underactuated robot hand}

\subsection{Kinematics analysis of the finger}

Since all the finger structures of the robot hand are identical, one of the fingers is represented as a kinematic analysis, and the remaining fingers are the same in the calculation method and process when calculating the kinematics model [39-40]. Corresponding mathematical operations can be performed to obtain the corresponding D-H change matrix:

$$
{ }_{i}^{i-1} T=\left[\begin{array}{cccc}
\cos \theta_{i} & -\sin \theta_{i} \cos \alpha_{i} & \sin \theta_{i} \sin \alpha_{i} & L_{i} \cos \theta_{i} \\
\sin \theta_{i} & \cos \theta_{i} \cos \alpha_{i} & -\sin \alpha_{i} \cos \theta_{i} & L_{i} \sin \theta_{i} \\
0 & \sin \alpha_{i} & \cos \alpha_{i} & d_{i} \\
0 & 0 & 0 & 1
\end{array}\right]
$$


130 where, $L_{i}$ indicates the length of each knuckle, where $i$ is the number of joints; $d_{i}$ indicates the axial

131 vertical distance of the two coordinate axes along the axis of rotation of adjacent bars; $\alpha_{i}$ indicates

132 the torsion angle of the corresponding knuckle; and $\theta_{i}$ indicates the angle between the opposite corner of the knuckle and the adjacent knuckle.

The D-H coordinate system of the modular finger is shown in Figure 5 where $\left(x_{1}, y_{1}, z_{1}\right)$ is the fixed reference frame coordinate and the rest is the dynamic reference system.

136

2 are respectively given in Eqs. (2) and (4).

$$
\begin{aligned}
{ }_{0}^{1} T & =\left[\begin{array}{cccc}
\cos \theta_{1} & -\sin \theta_{1} \cos \alpha_{1} & \sin \theta_{1} \sin \alpha_{1} & L_{i} \cos \theta_{1} \\
\sin \theta_{1} & \cos \theta_{1} \cos \alpha_{1} & -\sin \alpha_{1} \cos \theta_{1} & L_{1} \sin \theta_{1} \\
0 & 0 & 1 & 0 \\
0 & 0 & 0 & 1
\end{array}\right] \\
{ }_{1}^{2} T & =\left[\begin{array}{cccc}
\cos \theta_{1} & -\sin \theta_{1} \cos \alpha_{1} & \sin \theta_{1} \sin \alpha_{1} & L_{i} \cos \theta_{1} \\
\sin \theta_{1} & \cos \theta_{1} \cos \alpha_{1} & -\sin \alpha_{1} \cos \theta_{1} & L_{1} \sin \theta_{1} \\
0 & \sin \alpha_{1} & \cos \alpha_{1} & d_{1} \\
0 & 0 & 0 & 1
\end{array}\right]
\end{aligned}
$$

Therefore, one can be known from Eq. (1) that:

$$
{ }_{2}^{0} T={ }_{0}^{1} T \sqcup_{1}^{2} T={ }_{2}^{0} T=\left[\begin{array}{cccc}
C_{12} & -S_{12} & 0 & L_{1} C_{1}+L_{2} C_{12} \\
S_{12} & C_{12} & 0 & L_{1} S_{1}+L_{2} S_{12} \\
0 & 0 & 1 & 0 \\
0 & 0 & 0 & 1
\end{array}\right]
$$

143 where $S_{i}=\sin \theta_{\mathrm{i}}, C_{i}=\cos \theta_{\mathrm{i}}, S_{i j}=\sin \left(\theta_{\mathrm{i}}+\theta_{\mathrm{j}}\right), C_{i j}=\cos \left(\theta_{\mathrm{i}}+\theta_{\mathrm{j}}\right)$.

Then, the position of the fingertip coordinate system in the base coordinate system is as shown in Eq. (5). 


$$
\left[\begin{array}{l}
x_{g} \\
y_{g}
\end{array}\right]=\left[\begin{array}{l}
L_{1} C_{1}+L_{2} C_{12} \\
L_{1} S_{1}+L_{2} S_{12}
\end{array}\right]
$$

According to the previous pose transformation matrix of a single finger, the pose of the three

161 fingers in the base coordinate system can be obtained by:

The whole-hand kinematics was based on single-finger kinematics, the most important aspect of which is to find out how each finger coordinate system transforms the entire palm coordinate system. We first established the coordinate relationship shown in Figure 6, where the palm coordinate system is $\left\{O_{0} X_{0} Y_{0} Z_{0}\right\}$, and the coordinate system of the three fingers is $\left\{O_{1} X_{1} Y_{1} Z_{1}\right\},\left\{O_{2} X_{2} Y_{2} Z_{2}\right\}$, and $\left\{O_{3} X_{3} Y_{3} Z_{3}\right\}$, respectively.

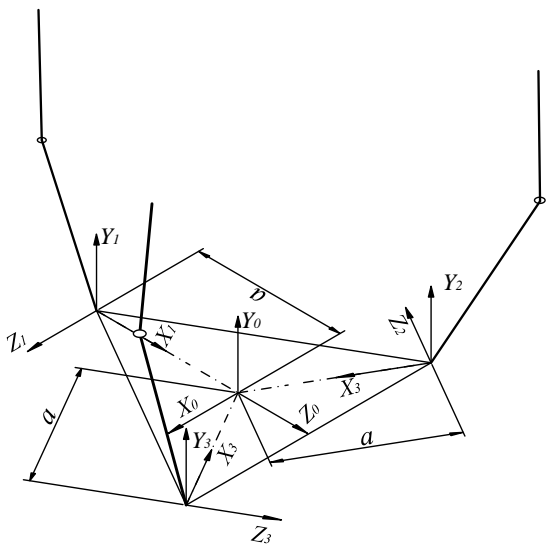

Figure 6. Schematic diagram of the palm coordinate system.

Figure 11 can be used to analyze the transformation of each finger coordinate system relative to the base coordinate system. Only the base coordinate system needs to be rotated along the $y$ axis, and then moved along the $x$ axis to obtain the corresponding finger coordinate system. The details are shown in Table 2 below.

Table 2. Transformation of finger coordinate system to base coordinate system.

\begin{tabular}{lcc}
\hline Name & Rotation angle along Y axis $\left(^{\circ}\right)$ & Moving distance along X direction $(\mathrm{mm})$ \\
\hline Finger 1 & 0 & $-a=37.5$ \\
Finger 2 & 120 & $-a=37.5$ \\
Finger 3 & -120 & $-a=37.5$ \\
\hline
\end{tabular}

According to Table 2, the pose of three fingers can be obtained in the palm coordinate system, as shown in Eq. (6):

$$
\begin{aligned}
{ }_{\mathrm{B}} T=\left[\begin{array}{cccc}
1 & 0 & 0 & -a \\
0 & 1 & 0 & 0 \\
0 & 0 & 1 & 0 \\
0 & 0 & 0 & 1
\end{array}\right] \quad{ }_{\mathrm{B}}^{E} T=\left[\begin{array}{cccc}
\cos \left(120^{\circ}\right) & 0 & \sin \left(120^{\circ}\right) & -a \\
0 & 1 & 0 & 0 \\
-\sin \left(120^{\circ}\right) & 0 & \cos \left(120^{\circ}\right) & 0 \\
0 & 0 & 0 & 1
\end{array}\right] \\
{ }_{\mathrm{B}}^{F} T=\left[\begin{array}{cccc}
\cos \left(-120^{\circ}\right) & 0 & \sin \left(-120^{\circ}\right) & -a \\
0 & 1 & 0 & 0 \\
-\sin \left(-120^{\circ}\right) & 0 & \cos \left(-120^{\circ}\right) & 0 \\
0 & 0 & 0 & 1
\end{array}\right]
\end{aligned}
$$




$$
\begin{gathered}
{ }_{B O}^{D} T={ }_{B}^{D} T \sqcup_{0}^{2} T=\left[\begin{array}{cccc}
C_{12} & -S_{12} & 0 & L_{1} C_{1}+L_{2} C_{12}-a \\
S_{12} & C_{12} & 0 & L_{1} S_{1}+L_{2} S_{12} \\
0 & 0 & 1 & 0 \\
0 & 0 & 0 & 1
\end{array}\right] \\
{ }_{B O}^{E} T={ }_{B}^{E} T \square_{0}^{2} T=\left[\begin{array}{cccc}
-\frac{1}{2} C_{12} & \frac{1}{2} S_{12} & \frac{\sqrt{3}}{2} & -\frac{1}{2}\left(L_{1} C_{1}+L_{2} C_{12}\right)-a \\
S_{12} & C_{12} & 0 & L_{1} S_{1}+L_{2} S_{12} \\
-\frac{\sqrt{3}}{2} C_{12} & \frac{\sqrt{3}}{2} S_{12} & -\frac{1}{2} & -\frac{\sqrt{3}}{2}\left(L_{1} C_{1}+L_{2} C_{12}\right) \\
0 & 0 & 0 & 1
\end{array}\right] \\
{ }_{B O}^{D} T={ }_{B}^{D} T \square_{0}^{2} T=\left[\begin{array}{cccc}
-\frac{1}{2} C_{12} & \frac{1}{2} S_{12} & -\frac{\sqrt{3}}{2} & -\frac{1}{2}\left(L_{1} C_{1}+L_{2} C_{12}\right)-a \\
S_{12} & C_{12} & 0 & L_{1} S_{1}+L_{2} S_{12} \\
\frac{\sqrt{3}}{2} C_{12} & -\frac{\sqrt{3}}{2} S_{12} & -\frac{1}{2} & \frac{\sqrt{3}}{2}\left(L_{1} C_{1}+L_{2} C_{12}\right) \\
0 & 0 & 0 & 1
\end{array}\right]
\end{gathered}
$$

where $S_{i}=\sin \theta_{\mathrm{i}}, C_{i}=\cos \theta_{\mathrm{i}}, S_{i j}=\sin \left(\theta_{\mathrm{i}}+\theta_{\mathrm{j}}\right), C_{i j}=\cos \left(\theta_{\mathrm{i}}+\theta_{\mathrm{j}}\right)$.

According to Eqs. (7)-(9), the motion space of the three-finger fingertip in the palm base coordinate system, as shown in Figure. 7(a), can be obtained by MATLAB programming, that is, the working space of the entire robot in the state of grasping the three-finger envelope. At the same time, the working space for calculating the different configurations of the claws is shown in Figure 7(b)(f).

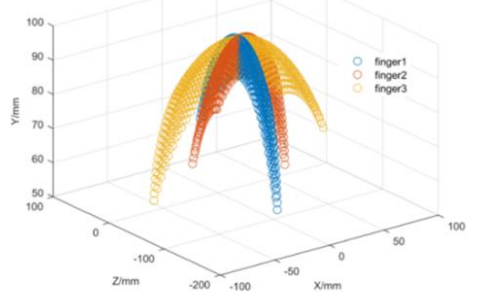

(a) Three-finger mode

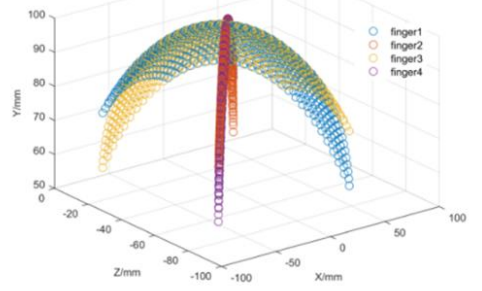

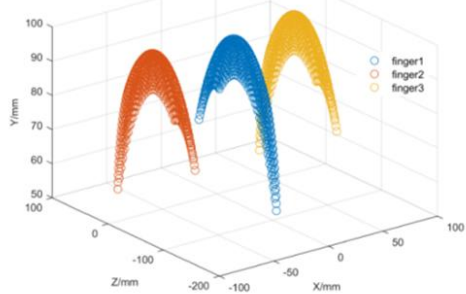

(b) Three-finger parallel mode

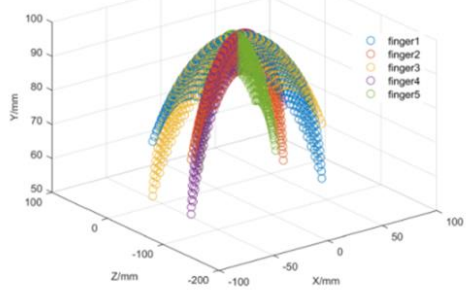

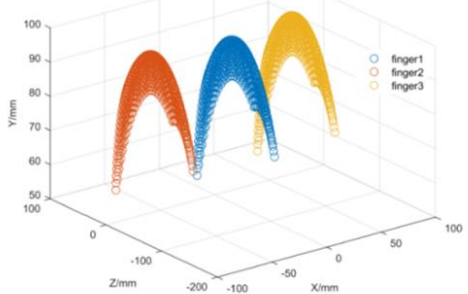

(c) Three-finger palm mode

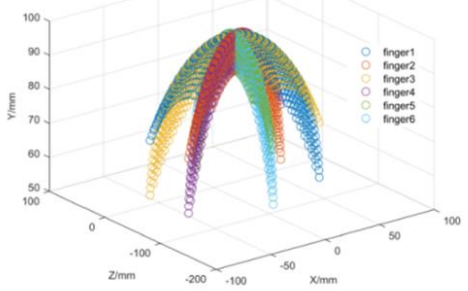


Through the static analysis of the finger, the relationship between the driving force and the contact force of each finger joint on the object can be obtained. In envelope grabbing, The contact force of each finger depends on the external constraints and the structure of the finger. The mechanism sketch of the mechanical finger is shown in Figure 8.
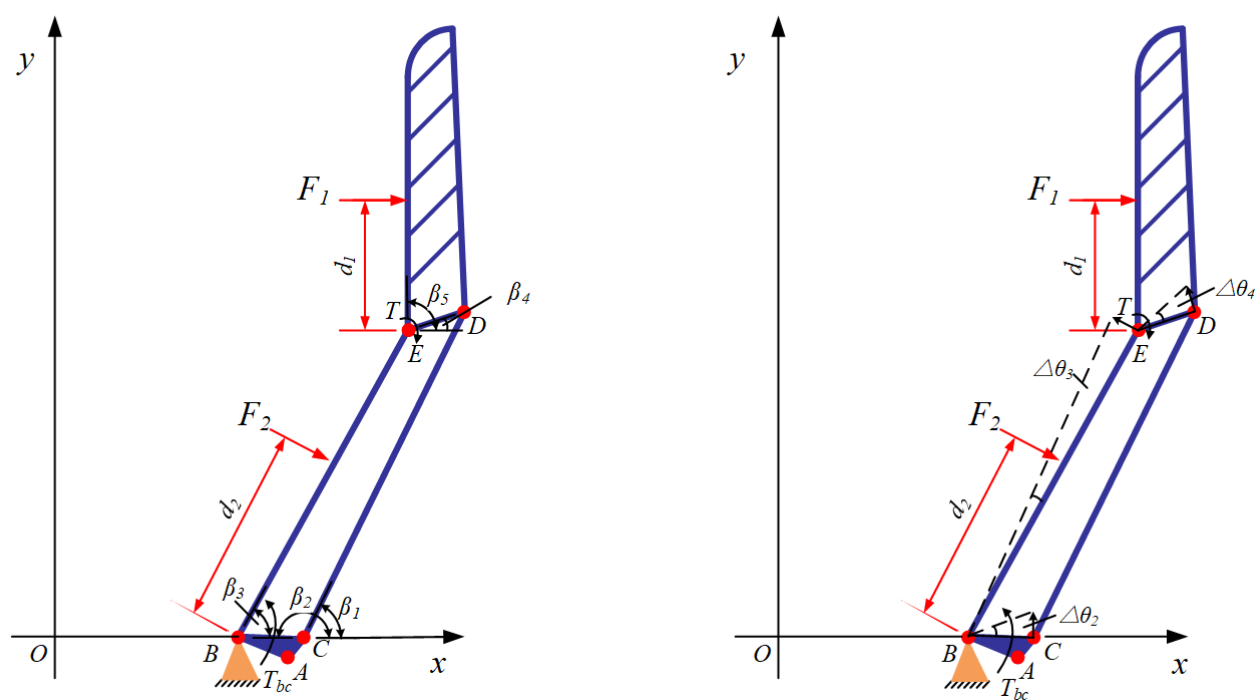

Figure8. Mechanism sketch of mechanical finger.

According to the principle of virtual work, we can get:

$$
\delta W=\sum_{i=1}^{n} F_{i} \square \delta_{r_{i}}=0
$$

After removing the binding force, the finger has a 2-DOF (degree of freedom) structure. We choose contact force $F_{1}$ and $F_{2}$, spring torque $T$ and driving moment $T_{b c}$ as generalized force. The angle of rotation of each connecting rod relative to the horizontal axis of the coordinate system is as follows: $\beta_{1} 、 \beta_{2} 、 \beta_{3}$,

$\beta_{4}, \beta_{5}$. Then the coordinates of each contact point are:

$$
\begin{aligned}
& \left\{\begin{array}{l}
x_{F_{2}}=x_{B}+d_{2} \cos \beta_{3} \\
y_{F_{2}}=d_{2} \sin \beta_{3}
\end{array}\right. \\
& \left\{\begin{array}{l}
x_{F_{2}}=x_{B}+l_{B E} \cos \beta_{3}-d_{1} \cos \beta_{5} \\
y_{F_{2}}=l_{B E} \sin \beta_{3}+d_{1} \sin \beta_{5}
\end{array}\right.
\end{aligned}
$$

Apply virtual corner to finger $\Delta \theta_{1} 、 \Delta \theta_{2} 、 \Delta \theta_{3}$, The virtual work done by contact force $F_{1}$ is:

$$
\delta W_{1}=F_{1} \cdot l_{B E} \cdot \cos \left(\beta_{3}-\beta_{5}\right) \cdot \Delta \theta_{3}+F_{1} \cdot d_{1} \cdot \Delta \theta_{5}
$$

The virtual work done by contact force $F_{2}$ is:

$$
\delta W_{2}=F_{2} \cdot d_{2} \cdot \Delta \theta_{3}
$$

The virtual work done by spring torsion $T$ is:

$$
\delta W_{T}=T \cdot\left(\Delta \theta_{5}-\Delta \theta_{3}\right)
$$

The virtual work principle equation of mechanical finger can be obtained by simultaneous equation Eq.15- Eq.17. 


$$
F_{1} \cdot l_{B E} \cdot \cos \left(\beta_{3}-\beta_{5}\right) \cdot \Delta \theta_{3}+F_{1} \cdot d_{1} \cdot \Delta \theta_{4}+F_{2} \cdot d_{2} \cdot \Delta \theta_{3}+T \cdot\left(\Delta \theta_{4}-\Delta \theta_{3}\right)-T_{B C} \cdot \Delta \theta_{2}=0
$$

187

188

$$
\left\{\begin{array}{l}
l_{B E} \sin \beta_{3} \Delta \theta_{3}+l_{D E} \sin \beta_{4} \Delta \theta_{4}=l_{B C} \sin \beta_{2} \Delta \theta_{2}+l_{C D} \sin \beta_{1} \\
l_{B E} \cos \beta_{3} \Delta \theta_{3}+l_{D E} \cos \beta_{4} \Delta \theta_{4}=l_{B C} \cos \beta_{2} \Delta \theta_{2}+l_{C D} \cos \beta_{1}
\end{array}\right.
$$

By simplifying equation 16 , equation 17 can be obtained.

$$
\Delta \theta_{2}=\frac{A \Delta \theta_{3}+B \Delta \theta_{4}}{C}
$$

189 In Equ.17.

$$
\begin{aligned}
& A=l_{B E} l_{D C} \sin \left(\beta_{3}-\beta_{1}\right) \\
& B=l_{D E} l_{D C} \sin \left(\beta_{4}-\beta_{1}\right) \\
& C=l_{B C} l_{D C} \sin \left(\beta_{2}-\beta_{1}\right)
\end{aligned}
$$

190

Plug equation 17 into equation 15:

$$
\left[F_{1} \cdot l_{B E} \cdot \cos \left(\beta_{3}-\beta_{5}\right)+F_{2} \cdot d_{2}-T-T_{B C} \cdot \frac{A}{C}\right] \cdot \Delta \theta_{3}+\left[F_{1} \cdot d_{1}+T-T_{B C} \cdot \frac{B}{C}\right] \cdot \Delta \theta_{4}=0
$$

191

Since Delta theta 3 and Delta theta 4 are linearly independent and independent, so:

$$
\left\{\begin{array}{l}
F_{1} \cdot l_{B E} \cdot \cos \left(\beta_{3}-\beta_{5}\right)+F_{2} \cdot d_{2}-T-T_{B C} \cdot \frac{A}{C}=0 \\
F_{1} \cdot d_{1}+T-T_{B C} \cdot \frac{B}{C}=0
\end{array}\right.
$$

192 A, B , C in Equation19 is the same as that in Equation17.

$$
\begin{aligned}
& A=l_{B E} l_{D C} \sin \left(\beta_{3}-\beta_{1}\right) \\
& B=l_{D E} l_{D C} \sin \left(\beta_{4}-\beta_{1}\right) \\
& C=l_{B C} l_{D C} \sin \left(\beta_{2}-\beta_{1}\right)
\end{aligned}
$$

193 On torsional spring, there is an equation:

$$
T=T_{0}+K_{T} \cdot\left(\beta_{5}-\beta_{3}\right)
$$

194 There are also equations for the geometric relationship of mechanical fingers.

$$
\left\{\begin{array}{l}
R \sin \beta_{3}=x_{B}+d_{2} \cos \beta_{3} \\
R \sin \beta_{5}=x_{B}+l_{b e} \cos \beta_{3}+d_{1} \cos \beta_{5}
\end{array}\right.
$$

If the value of contact force is required, the grasping state of the robot hand needs to be analyzed.

196 The static analysis of the grasping process is shown in Figure 9. 


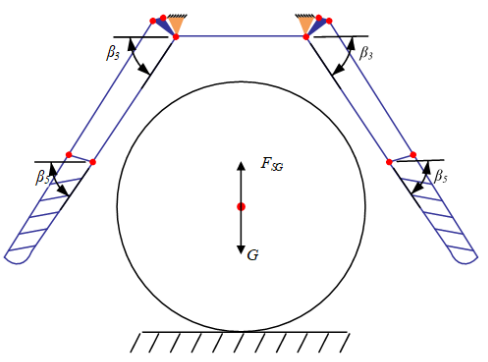

(a)

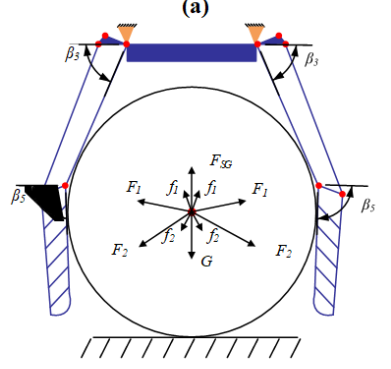

(d)

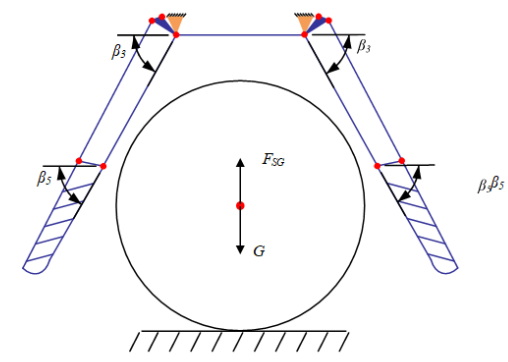

(b)

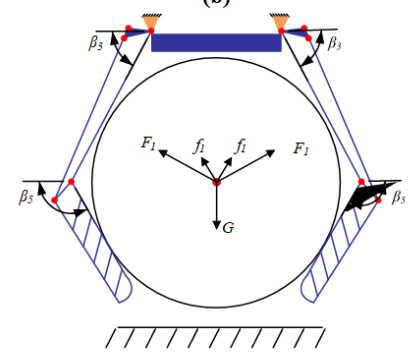

(e)

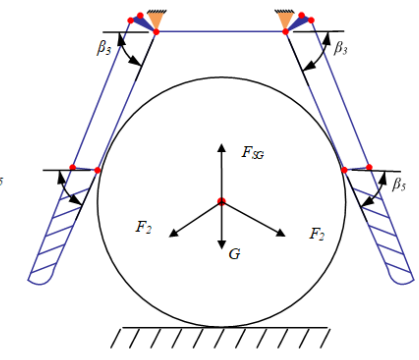

(c)

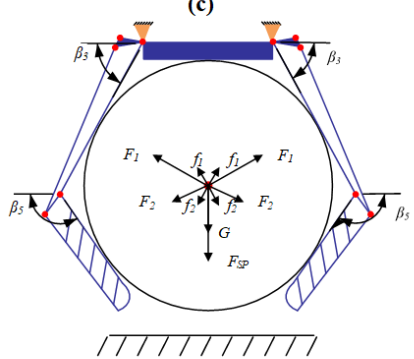

(f)

Figure 9. Force analysis of robot hand during grasping process.

When the robot hand at a lower speed, the grasping process can be equivalent to always in equilibrium, and the contact forces $F_{1}$ and $F_{2}$ can be solved in arbitrary grasping state. Because every instantaneous robot hand is grabbing the object, the friction between the object and the knuckle should also be considered.

In Figures 9 (a) and (b), the finger does not touch the object, and the contact forces $F_{1}$ and $F_{2}$ are 0.

The force acting on the object is:

$$
F_{S G}=G
$$

In Figure 9 (c), there is only contact force $F_{2}$, and the robot hand has no lifting action, so there is no friction. The force (vertical direction) equation of the object is as follows:

$$
F_{S G}=n F_{2} \cos \beta_{3}+G
$$

At this time, the contact force $\mathrm{F}_{2}$ can be obtained only by measuring the rotation angle of each finger joint and the ground support force $F_{S G}$ of the object.

In Figure $9(\mathrm{~d})$, there are both contact forces $F_{1}$ and $F_{2}$, and the friction produced by contact force $F_{1}$ is used to lift the object, and the friction produced by contact force $\mathrm{F}_{2}$ is resistance. Forces on an object:

$$
F_{S G}+n F_{1}\left(-\cos \beta_{5}\right)+n \mu F_{1} \sin \beta_{5}=n F_{2} \cos \beta_{3}+G+n \mu F_{2} \sin \beta_{3}
$$

In conjunction with the previous equations, we can get:

$$
\left\{\begin{array}{l}
F_{1} \cdot l_{B E} \cdot \cos \left(\beta_{3}-\beta_{5}\right)+F_{2} \cdot d_{2}-T-T_{B C} \cdot \frac{A}{C}=0 \\
F_{1} \cdot d_{1}+T-T_{B C} \cdot \frac{B}{C}=0 \\
F_{S G}+n F_{1}\left(-\cos \beta_{5}\right)+n \mu F_{1} \sin \beta_{5}=n F_{2} \cos \beta_{3}+G+n \mu F_{2} \sin \beta_{3} \\
T=T_{0}+K_{T} \cdot\left(\beta_{5}-\beta_{3}\right) \\
R \sin \beta_{3}=x_{B}+d_{2} \cos \beta_{3} \\
R \sin \beta_{5}=x_{B}+l_{b e} \cos \beta_{3}+d_{1} \cos \beta_{5}
\end{array}\right.
$$

At this time, the contact force $F_{1}$ and $F_{2}$ can be obtained only by measuring the rotation angle of each finger joint and the ground support force $\mathrm{F}_{\mathrm{SG}}$ of the object.

In Figure 9 (e), the contact force F1 begins to grasp the object close to the palm, and the friction force $\mathrm{F} 1$ is resistance, which satisfies the equation:

$$
n F_{1}\left(-\cos \beta_{5}\right)-n \mu F_{1} \sin \beta_{5}=G
$$


At this time, the contact force $\mathrm{F}_{1}$ can be obtained only by measuring the rotation angle of each finger joint and the gravity of the object.

In Figure 9 (f), the object is in equilibrium at this time, and the object does not move relative to the robot hand, so there is no friction force. The force on the object is as follows:

$$
n F_{1}\left(-\cos \beta_{5}\right)=n F_{2} \cos \beta_{3}+G+F_{S P}
$$

In conjunction with the previous equations, we can get:

$$
\left\{\begin{array}{l}
F_{1} \cdot l_{B E} \cdot \cos \left(\beta_{3}-\beta_{5}\right)+F_{2} \cdot d_{2}-T-T_{B C} \cdot \frac{A}{C}=0 \\
F_{1} \cdot d_{1}+T-T_{B C} \cdot \frac{B}{C}=0 \\
n F_{1}\left(-\cos \beta_{5}\right)=n F_{2} \cos \beta_{3}+G+F_{S P} \\
T=T_{0}+K_{T} \cdot\left(\beta_{5}-\beta_{3}\right) \\
R \sin \beta_{3}=x_{b}+d_{2} \cos \beta_{3} \\
R \sin \beta_{5}=x_{b}+l_{b e} \cos \beta_{3}+d_{1} \cos \beta_{5}
\end{array}\right.
$$

At this time, the contact forces $F_{1}$ and $F_{2}$ can be obtained only by measuring the rotation angle of each finger joint, the supporting force $F_{\mathrm{SP}}$ of the ground and the gravity of the object.

In conclusion, the contact force in the process of grasping robot hand can be calculated by measuring joint rotation angle and ground support force. In the actual grasping process, the factors such as contact material, contact deformation, damping and so on should be taken into account. The contact force changes in the grasping process of the robot hand will be comprehensively analyzed by the multi-lift dynamics simulation software.

\section{Dynamics simulation analysis of robot hand grasping process}

In this paper, multi-body dynamics software ADAMS is used to simulate and analyze the three-finger Heart Mode, three-finger parallel mode and six-finger Heart mode of the robot hand. The characteristic curves and contact force of each finger joint in the process of grasping are obtained, and the theoretical model is verified. The target is a rigid sphere with radius $R=50 \mathrm{~mm}$ and gravity $\mathrm{G}=19.6 \mathrm{~N}$.

Before grasping simulation, the contact force between the finger and the grasping target of the robot hand should be set. In ADAMS, the IMPACT function method is used to define the contact force, and the friction force between objects is considered. Contact force settings are shown in Table 3 .

Table 3. parameters of each finger contact force.

\begin{tabular}{lll}
\hline Contact force parameters & Second knuckle & Third knuckle \\
\hline materials & Steel and aluminium & Steel and rubber \\
Stiffness coefficient/ $(\mathrm{N} / \mathrm{mm})$ & 35000 & 2855 \\
Force Exponent & 1.5 & 1.1 \\
Damping/ (Ns/mm) & 28 & 0.57 \\
Penetration Depth & 0.1 & 0.1 \\
Static friction coefficient & 0.25 & 0.25 \\
Dynamic friction coefficient & 0.2 & 0.2 \\
\hline
\end{tabular}




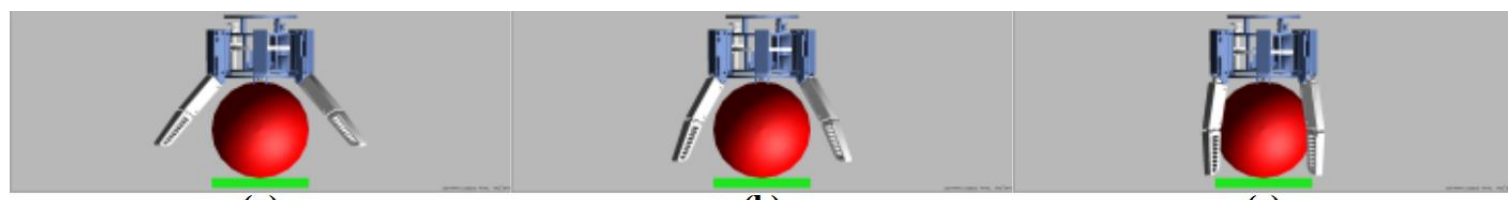

(a)

(b)

(c)
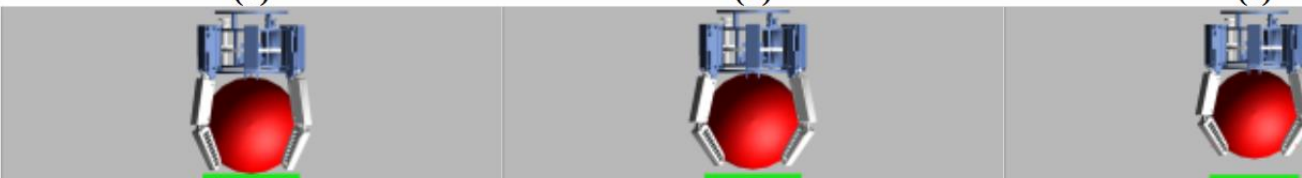

Figure 10. The process of grabbing objects in Three-fingered Heart Mode.

The angular displacement curves of the second and third knuckles are shown in Figure 11.a and the contact force of the second and third knuckles are shown in Figure 11.b. Because the underactuated robot hand are symmetrical, the contact force and angular displacement curves of the three fingers are the same, so one of them is selected for analysis.

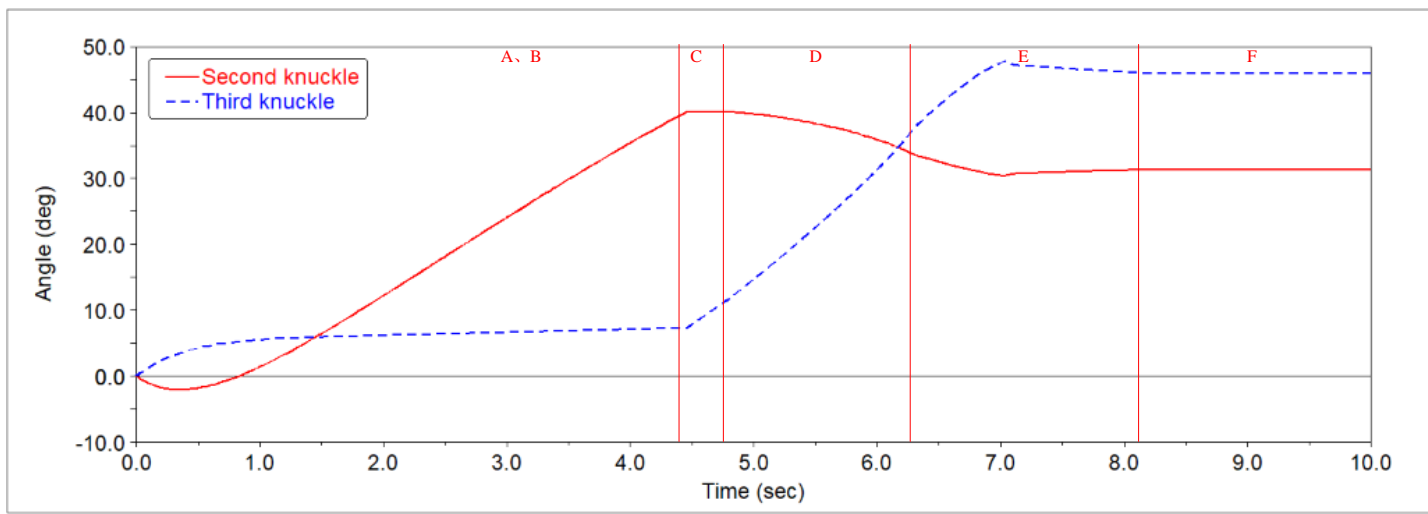

(a) Angular displacement curves of the second and third fingers

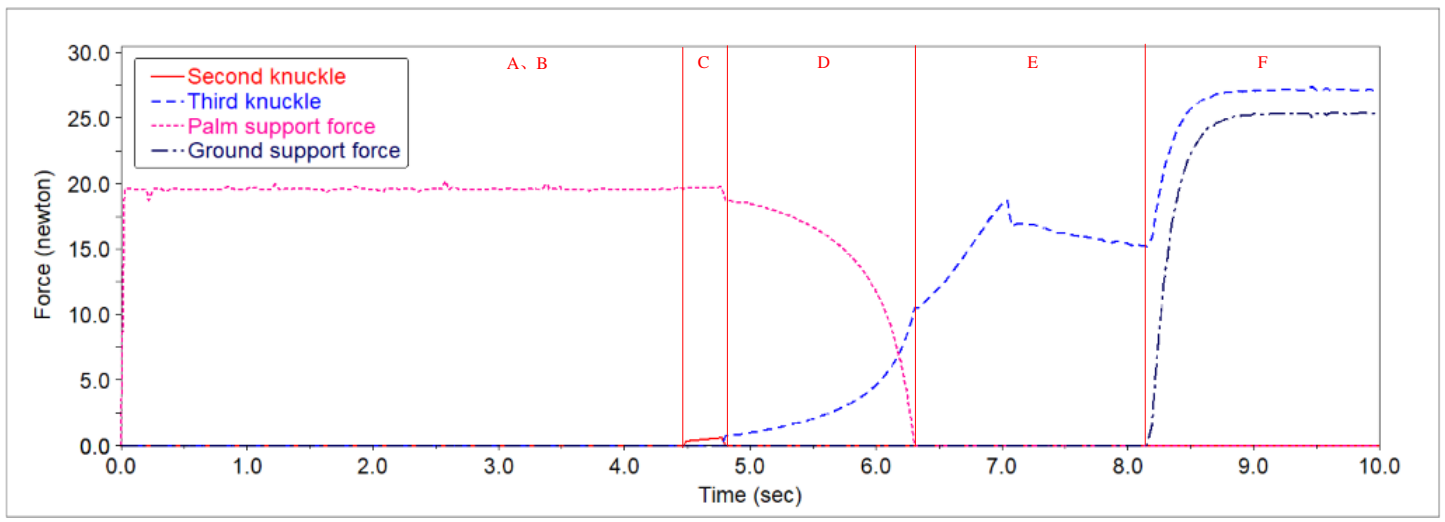

(b) Contact forces of the second and third knuckles

Figure 11. Characteristic curve of Three-fingered Heart Mode

In order to verify the validity of the static model in the previous section, a random set of numerical values under four states is taken for calculation in the process of grasping the robot hand. The results are shown in Table 4.

Table 4. Comparison table of simulation analysis and theoretical analysis in Three-fingered Heart Mode

\begin{tabular}{|c|c|c|c|c|}
\hline Operating state of robot hand & C & D & E & F \\
\hline time & 4.7764 & 6.2 & 8 & 10 \\
\hline Second knuckle swing angle & 40.2414 & 34.5424 & 31.3628 & 31.5035 \\
\hline
\end{tabular}




\begin{tabular}{|c|c|c|c|c|}
\hline Third knuckle swing angle & 11.4072 & 35.3133 & 46.2652 & 45.9919 \\
\hline Ground support force & 19.7691 & 6.3345 & 0 & 0 \\
\hline Palm support force & 0 & 0 & 0 & 25.3979 \\
\hline $\begin{array}{l}\text { Simulation value of Contact force of second } \\
\text { knuckle }\end{array}$ & 0.6233 & 0 & 0 & 0 \\
\hline Simulation value of Contact force of third knuckle & 0 & 7.3308 & 15.444 & 27.1728 \\
\hline Calculated value of contact force of second knuckle & 0.679 & 0 & 0 & 0 \\
\hline Calculated value of contact force of third knuckle & 0 & 6.8327 & 17.67 & 27.9212 \\
\hline Error & $8.00 \%$ & $6.70 \%$ & $14.00 \%$ & $2.60 \%$ \\
\hline
\end{tabular}

\subsection{Three-finger Parallel Grab Mode}

The process of grasping cylinder of robot hand in parallel grasping mode is simulated as shown in Figure 12.

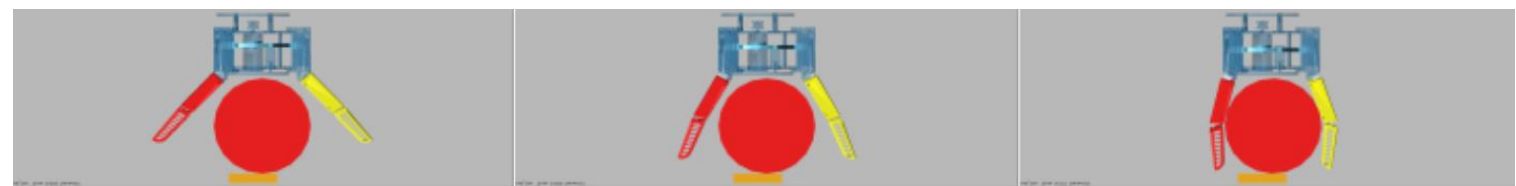

(a)

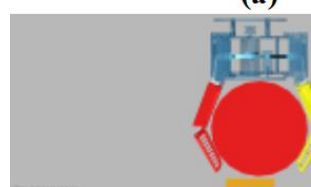

(d) (b)

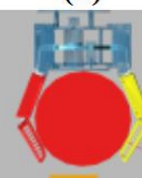

(e) (c)

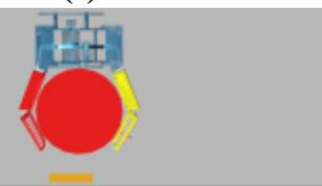

(f)

Figure12. The process of grabbing objects in Three-finger Parallel Mode

The simulation data are analyzed by ADAMS post-processing module. The angular displacement curves of the second and third knuckles are shown in Figure 13.a and the contact force of the second and third knuckles are shown in Figure 13.b. Because the number of fingers on both sides of the robot hand is not equal in parallel grasping mode (There are two fingers on one side and one finger on the other.), the data of one finger from left to right are compared and analyzed.

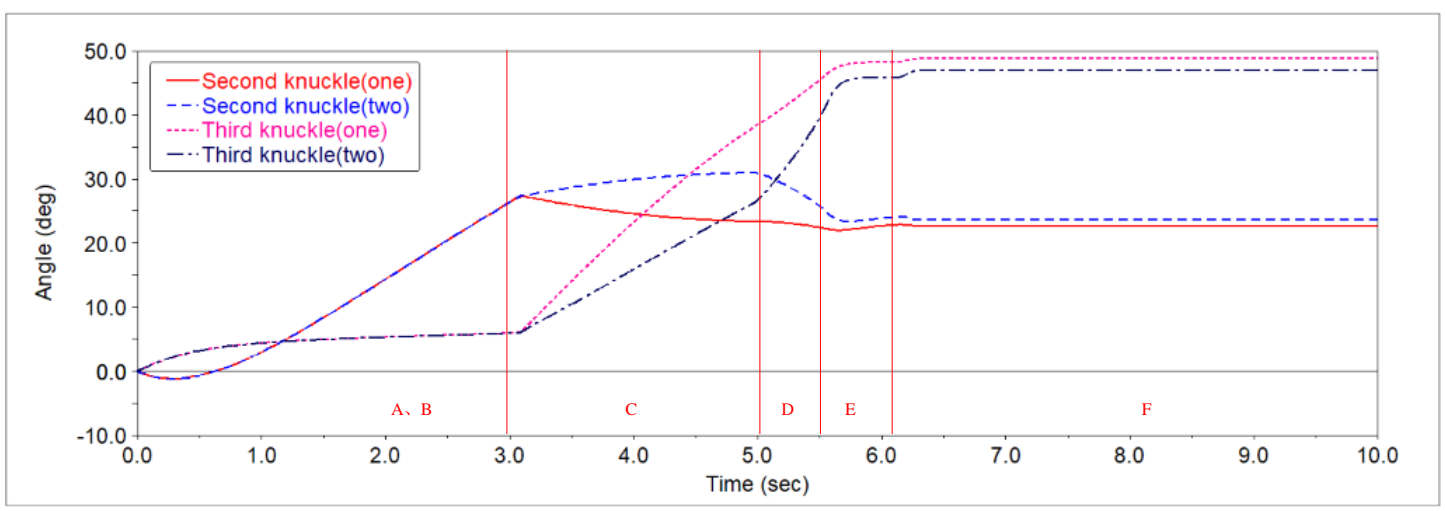

(a) Angular displacement curves of the second and third fingers. 


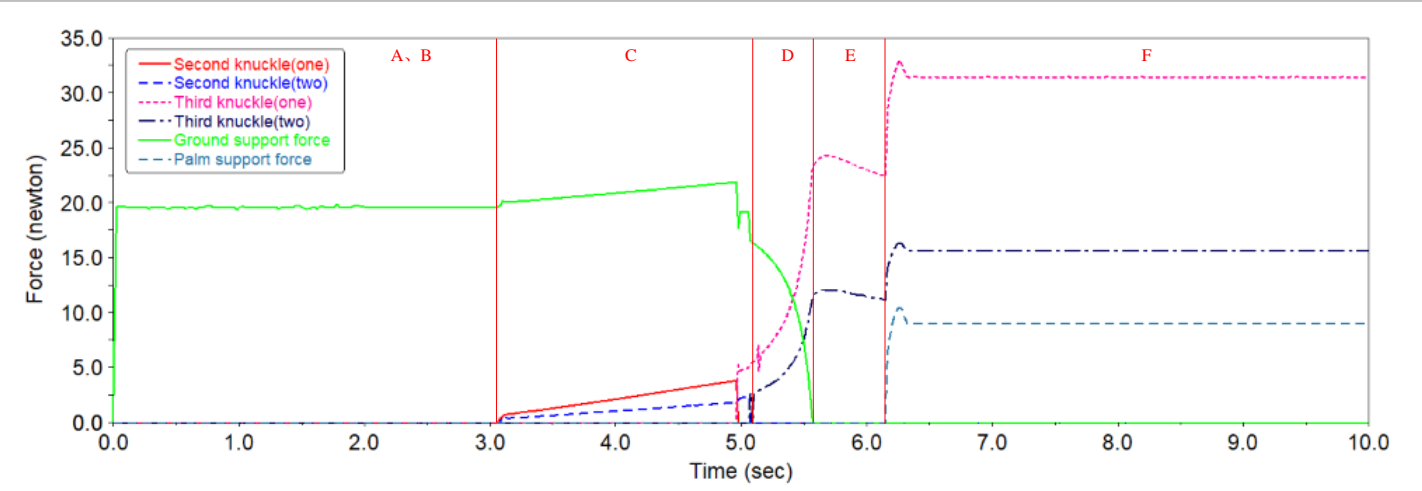

(b) Contact forces of the second and third knuckles.

Figure13. Characteristic curve of three-fingered parallel mode.

In order to verify the validity of the static model in the previous section, a random set of numerical values under four states is taken for calculation in the process of grasping the robot hand. The results are

Table 5. Comparison table of simulation analysis and theoretical analysis in three-fingered parallel mode.

\begin{tabular}{|c|c|c|c|c|c|c|c|c|}
\hline Operating state of robot hand & \multicolumn{2}{|c|}{$\mathbf{C}$} & \multicolumn{2}{|c|}{$\mathbf{D}$} & \multicolumn{2}{|c|}{$\mathbf{E}$} & \multicolumn{2}{|c|}{$\mathbf{F}$} \\
\hline time & \multicolumn{2}{|c|}{4.9642} & \multicolumn{2}{|c|}{5.2891} & \multicolumn{2}{|c|}{5.6332} & \multicolumn{2}{|c|}{10} \\
\hline Second knuckle swing angle & 23.455 & 31.073 & 23.077 & 28.428 & 22.084 & 23.564 & 22.789 & 23.681 \\
\hline Ground support force & \multicolumn{2}{|c|}{21.9013} & \multicolumn{2}{|c|}{14.0854} & \multicolumn{2}{|c|}{0} & \multicolumn{2}{|c|}{0} \\
\hline Palm support force & \multicolumn{2}{|c|}{0} & \multicolumn{2}{|c|}{0} & \multicolumn{2}{|c|}{0} & \multicolumn{2}{|c|}{9.0459} \\
\hline Simulation value of Contact force of second knuckle & 3.826 & 1.8351 & 0 & 0 & 0 & 0 & 0 & 0 \\
\hline Simulation value of Contact force of third knuckle & 0 & 0 & 8.0293 & 3.8692 & 24.107 & 12.000 & 31.473 & 15.669 \\
\hline error & $0.6 \%$ & $0.5 \%$ & $16.5 \%$ & $13.9 \%$ & $31.6 \%$ & $27.7 \%$ & $3.5 \%$ & $3.1 \%$ \\
\hline
\end{tabular}

4.3 Six-fingered Heart Mode

The six-finger heart-to-heart mode capture process is shown in Figure 14.

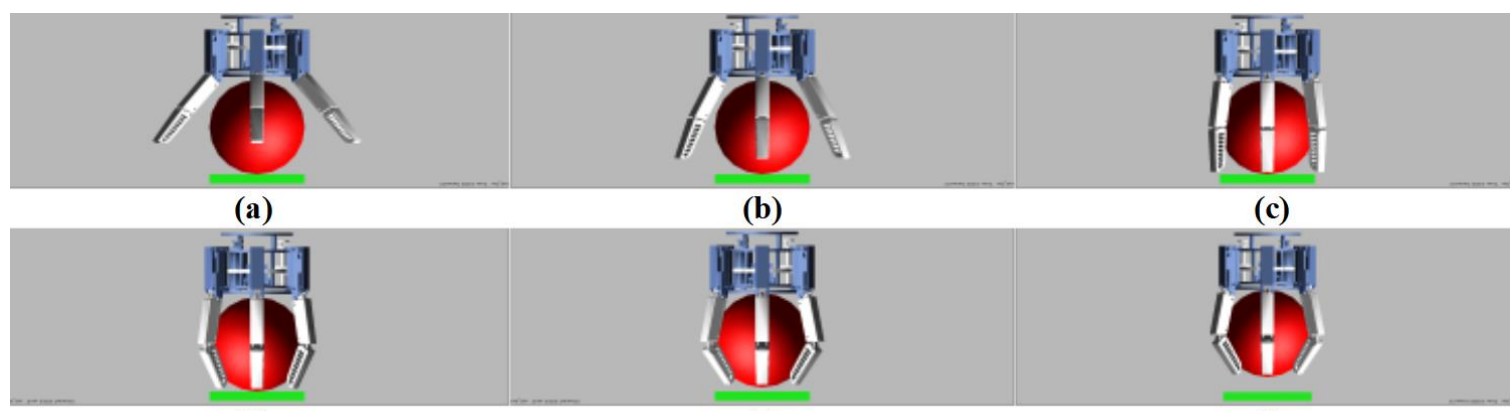

(d)

(e)

(f)

Figure 14. The process of grabbing objects in Six-fingered Heart Mode. 
The simulation data are analyzed by ADAMS post-processing module. The angular displacement curves of the second and third knuckles are shown in Figure15.a and the contact force of the second and third knuckles are shown in Figure 15.b. Because the underactuated robot fingers are symmetrically placed, the contact force and angular displacement curves of the six fingers are the same, so one of them is selected for analysis.

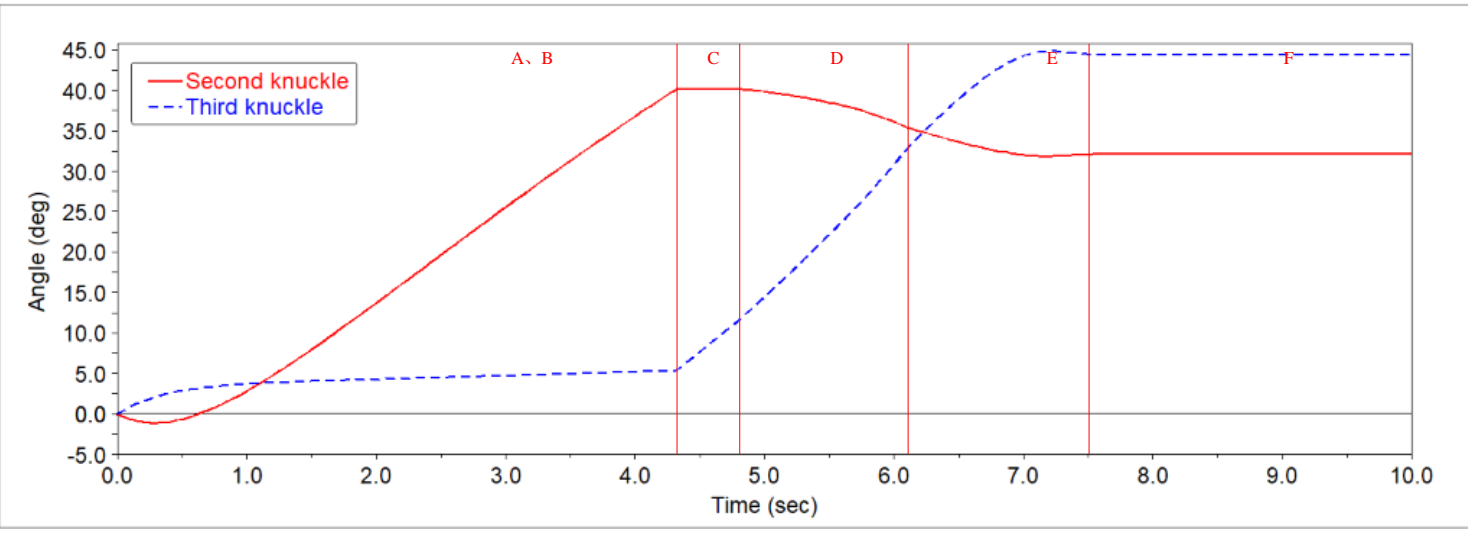

(a) Angular displacement curves of the second and third fingers

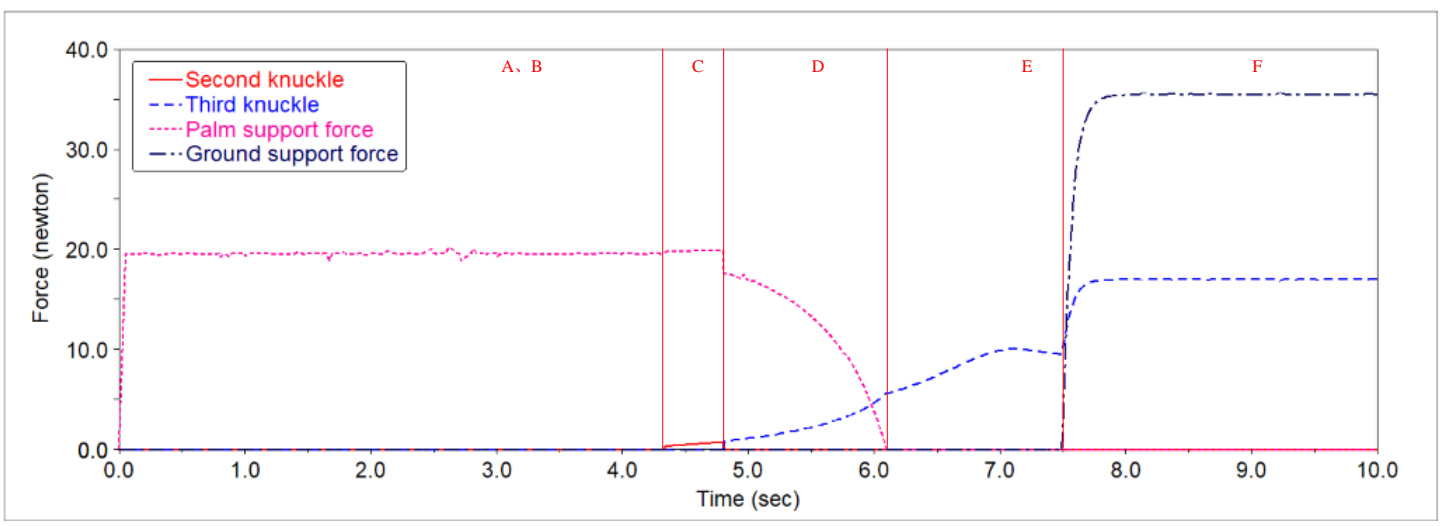

(b) Contact forces of the second and third knuckles

Figure 15. Characteristic curve of six-fingered heart mode

In order to verify the validity of the static model in the previous section, a random set of numerical values under four states is taken for calculation in the process of grasping the robot hand. The results are shown in Table 6.

Table 6. Comparison table of simulation analysis and theoretical analysis in six-fingered heart mode

\begin{tabular}{|c|c|c|c|c|}
\hline Operating state of robot hand & C & D & E & F \\
\hline time & 4.8 & 5.8 & 7.4 & 10 \\
\hline Second knuckle swing angle & 40.2409 & 37.2725 & 32.0832 & 10 \\
\hline Third knuckle swing angle & 11.5202 & 27.1133 & 44.6789 & 32.1903 \\
\hline Ground support force & 19.9984 & 9.0452 & 0 & 0 \\
\hline Palm support force & 0 & 0 & 0 & 35.4912 \\
\hline Simulation value of Contact force of second knuckle & 0.7698 & 0 & 0 & 0 \\
\hline Simulation value of Contact force of third knuckle & 0 & 3.3721 & 9.6837 & 17.6286 \\
\hline Calculated value of contact force of second knuckle & 0.8009 & 0 & 0 & 0 \\
\hline Calculated value of contact force of third knuckle & 0 & 3.379 & 9.2647 & 17.5522 \\
\hline Error & $4.0 \%$ & $0.2 \%$ & $-4.3 \%$ & $-0.4 \%$ \\
\hline
\end{tabular}


Through the simulation analysis of three grasping modes, the following problems can be found by comparing the error between the theoretical value and the simulation value of contact force.

(1) Excessive impact results in large deformation of spring and affects grasping stability.

(2) The impact size is related to the motion function of the actuator. If the speed of the actuator is reduced when it approaches the target, the impact can be significantly reduced.

(3) When calculating friction force, it is necessary to consider the motion state of the object.

(4) Because of the different number of knuckles on both sides of the parallel grasp mode, the object oscillates during the grasp process. The friction between the object and the ground should be considered in the theoretical calculation.

(5) The movement process of the fingers on both sides of parallel grasping mode is not uniform. The friction may occur when one side is lifting force and the other side is resisting force.

\section{Grasping experiment of modular robot hand}

The physical grasping experiment of the modular underactuated robot hand consisted of two parts: (1) The first is the experiment of mechanical finger in the mode of center-of-mind arrangement. The purpose is to verify the working state of the robot hand when it grasps a similar sphere. (2) The second is the experiment of mechanical finger in the mode of parallel arrangement. The purpose is to verify the working state of the robot hand when grasping long strip shape objects. The state after the robot had finished grabbing is shown in Figure 16.

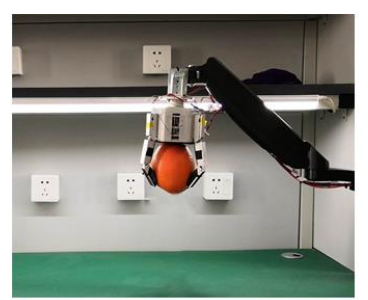

(a)

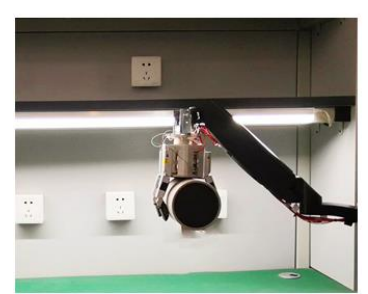

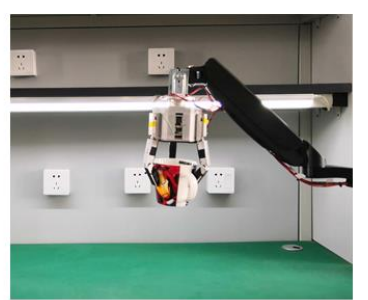

(b)

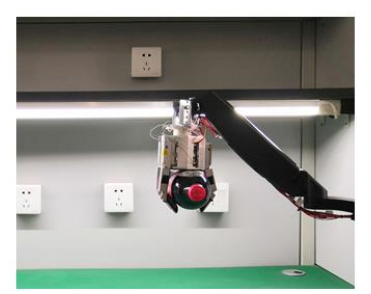

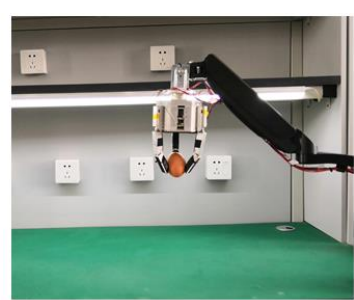

(c)

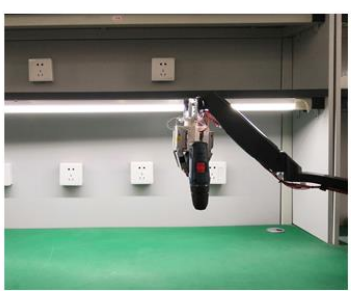

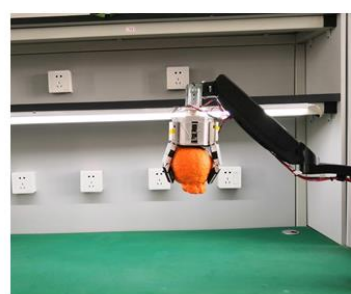

(d)

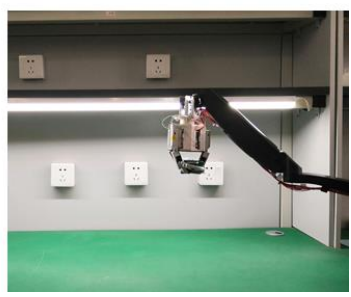

Figure 16. Robot hand grabbing experiments: (a) oranges; (b) cup; (c) egg; (d) big orange ; (e) tea cans ; (f) bottle; (g) electrodrill; (h) Hexagon wrench.

During the experiment, the fingertip hollow flexible rubber worked as a good buffer. The film pressure sensor worked normally and could feed the contact force back to the control system. The rotation angle of knuckles, the deformation of fingertip rubber and the center of mass of the irregular object will affect the collected data when the robot hand grabs the irregular objects, and the data of multiple grabs is not regular, So we choose a few regular grabbing data for analysis. The value of the contact force during the gripping process of the robot is shown in Figure 17. The driving torque of the motor is shown in Figure 18 


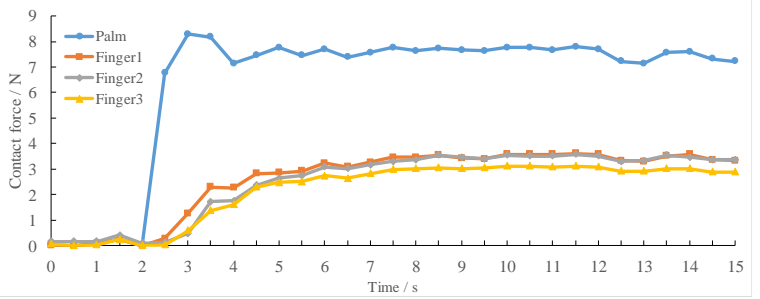

(a)

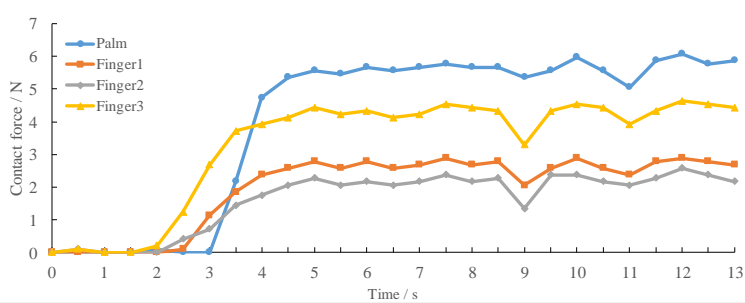

(c)

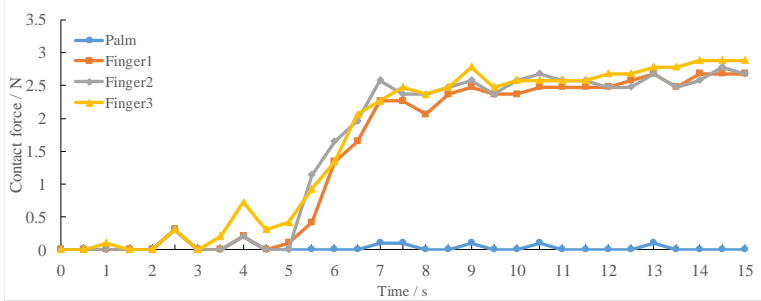

(b)

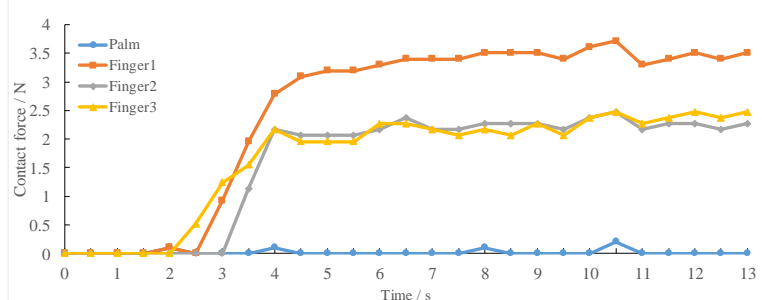

(d)

Figure 17. Experimental contact force curve: (a) orange; (b) cup; (c)tea cans; (d) bottle.

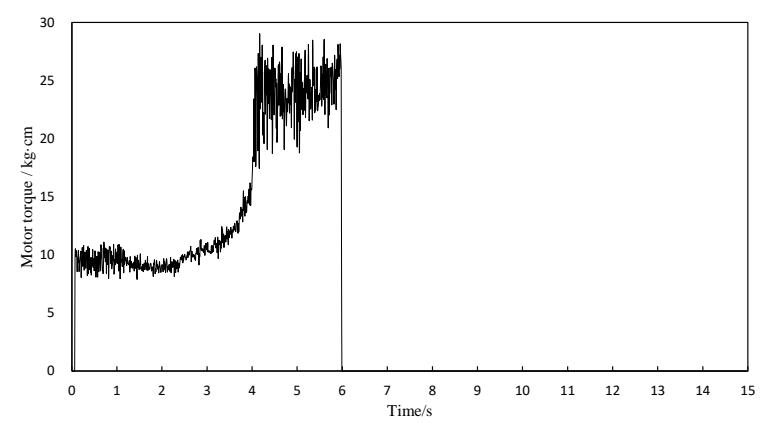

(a)

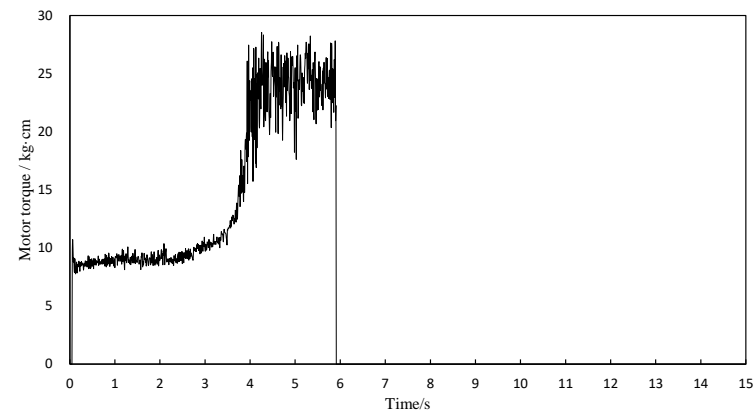

(c)

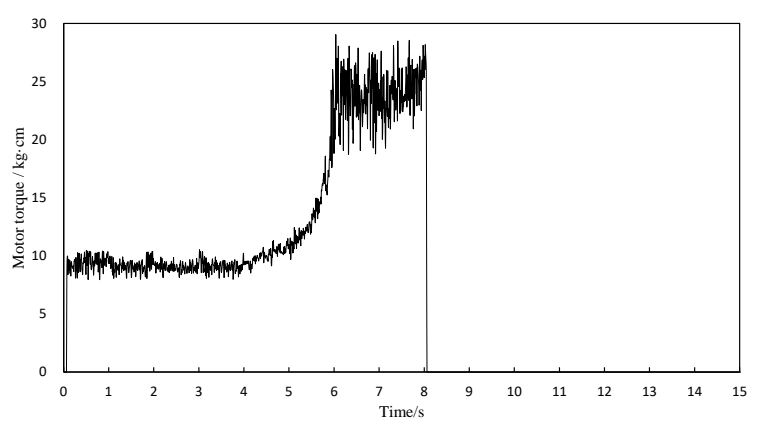

(b)

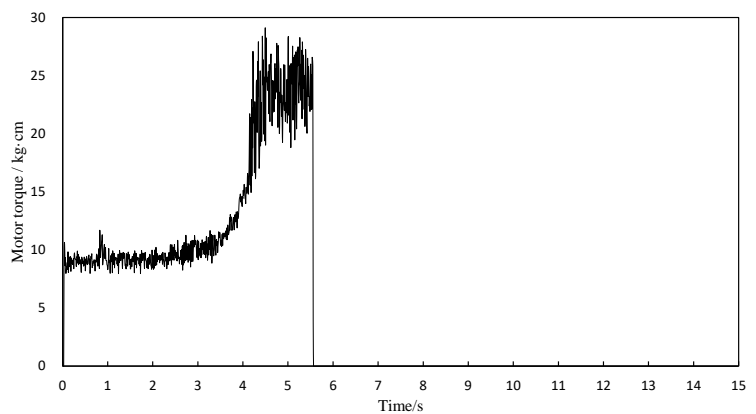

(d)

Figure 18. Experimental motor torque curve:
(a) orange; (b) cup; (c) tea cans; (d) bottle.

The experiments showed that the robot hand could adapt well to objects of different shapes and capture a variety of objects. In the experiment, the sensor in the middle of the palm was stable, and the contact force of the three knuckles was basically equal when in centripetal mode. When in parallel mode, the force of the left and right fingers was about double, and the contact force value was basically consistent with the simulation result. The robot hand can grasp objects according to the contact force feedback value, and control the grasping process to enhance its adaptability to various objects.

\section{Conclusions}

A modular underactuated multi-finger robot hand with a variable configuration was designed for the actual functional requirements of the mechanical gripper. The mathematical model of the finger 
was established according to the D-H method. The forward kinematics analysis was carried out on the robot hand, and the working space of the robot hand was simulated and verified. Further, a system analysis of the robot grabbing process was carried out, and the contact force during the gripping process of the robot hand was analyzed. Then, a virtual prototype model of the robot hand was constructed to verify the rationality of the structural design and calculate the key data based on ADAMS. Finally, the test platform of the modular underactuated multi-finger robot hand was built and the grabbing experiment was carried out. Experiments showed that the robot hand was designed reasonably to meet the gripping requirements of a variety of objects.

344 Featured Application: The modular underactuated multi-finger robot hand designed in this paper can meet various grasping requirements. It can flexibly change the configuration and layout according to the actual work needs. It is very suitable for the work needs of intelligent factories and robotic terminals.

Funding: This work was supported by the NSFC (61763036 and 5197090691), Important Foundation of Inner Mongolia University of Technology (ZD201701) and Australia ARC DECRA (DE190100931).

Conflicts of Interest: The authors declare no conflicts of interests.

\section{References}

[1] Mouri, T.; Endo, T.; Kawasaki, H.. Review of Gifu Hand and Its Application. Mechanics Based Design of Structures and Machines, 2011, 39: 210-228.

[2] Mouri, T.; Kawasaki, H.; Yoshikawa, K.; Takai, J.; Ito, S.. Anthropomorphic Robot Hand: Gifu Hand III. Robotics Society of Japan, 2004, 22, 55 -56.

[3] Chalon, M.; Wedler, A.; Baumann, A.; Bertleff, W.; Beyer, A.; Butterfaß, J.; Grebenstein, M.; Gruber, R.; Hacker, F.; Kraemer, E.. Dexhand: A Space qualified multi-fingered robotic hand. In Proceedings of 2011 IEEE International Conference on Robotics and Automation, 9-13 May 2011; pp. 2204-2210.

[4] Diftler, M. A.; Mehling, J. S.; Abdallah, M. E.; Radford, N. A.; Bridgwater, L. B.; Sanders, A. M.; Askew, R. S.; Linn, D. M.; Yamokoski, J. D.; Permenter, F. A.. Robonaut 2-The first humanoid robot in space. In Proceedings of 2011 IEEE International Conference on Robotics and Automation, 9-13 May 2011; pp. 2178-2183.

[5] Diftler, M. A.; Ahlstrom, T. D.; Ambrose, R. O.; Radford, N. A.; Joyce, C. A.; Pena, N. D. L.; Parsons, A. H.; Noblitt, A. L.. Robonaut 2-Initial activities on-board the ISS. In Proceedings of 2012 IEEE Aerospace Conference, 3-10 March 2012; pp. 1-12.

[6] Liu, H.; Wu, K.; Meusel, P.; Seitz, N.; Hirzinger, G.; Jin, M.H.; Liu, Y.W.; Fan, S.W.; Lan, T.; Chen, Z.P. Multisensory five-finger dexterous hand: The DLR/HIT Hand II. In Proceedings of 2008 IEEE/RSJ International Conference on Intelligent Robots and Systems, 22-26 Sept. 2008; pp. 3692-3697.

[7] Liu,Y.; JIN, M.; FAN, S.; LAN, T.; CHEN, Z.. Five-finger Dextrous Robot Hand DLR/HIT Hand II. Chinese Journal of Mechanical Engineering, 2009, 45: 10-17.

[8] Fruetel, J. A.; Renzi, R. F.; VanderNoot, V. A.; Stamps, J.; Horn, B. A.; West, J. A.; Ferko, S.; Crocker, R.; Bailey, C. G.; Arnold, D.; Wiedenman, B.. Microchip separations of protein biotoxins using an integrated hand - held device. Electrophoresis. 2005, 26(6): 1144-1154.

[9] Kochan, A.. Shadow delivers first hand. Industrial Robot: An International Journal. 2005, 32(1): 15-16. 
[10] Luo, C.; Yang, S.; Zhang, W.; Ren, Z.; Liang, J.. MPJ Hand: A self-adaptive underactuated hand with flexible fingers of multiple passive joints. In Proceedings of 2016 International Conference on Advanced Robotics and Mechatronics (ICARM), 18-20 Aug. 2016; pp. 184-189.

[11] Che, D.; Zhang, W.. GCUA Humanoid Robotic Hand with Tendon Mechanisms and Its Upper Limb. International Journal of Social Robotics, 2011, 3: 395-404.

[12] Song, S.; Zhang, W.. PCSS Hand: An Underactuated Robotic Hand with a Novel Parallel-Coupled Switchable Self-adaptive Grasp. Cham; pp. 481-491.

[13] Liang, D.; Zhang, W.. Parameters Optimization and Stability Analysis for a Parallel and Self-adaptive Underactuated Hand. Robot, 2017, 39: 282-291.

[14] Atasoy, A.; Kaya, E.; Toptas, E.; Kuchimov, S.; Kaplanoglu, E.; Ozkan, M.. 24 DOF EMG controlled hybrid actuated prosthetic hand. In Proceedings of 2016 38th Annual International Conference of the IEEE Engineering in Medicine and Biology Society (EMBC), 16-20 Aug. 2016; pp. 5059-5062.

[15] Odhner, L. U.; Ma, R. R.; Dollar, A. M.. Exploring Dexterous Manipulation Workspaces with the iHY Hand. Journal of the Robotics Society of Japan, 2014, 32: 318-322.

[16] Kobayashi, A.; Yamaguchi, K.; Kinugawa, J.; Arai, S.; Hirata, Y.; Kosuge, K.. Analysis of power grip force for uGRIPP. The Proceedings of JSME annual Conference on Robotics and Mechatronics (Robomec) 2017, 2017, 2P1-C09,

[17] Santina, C. D.; Piazza, C.; Grioli, G.; Catalano, M. G.; Bicchi, A.. Toward Dexterous Manipulation With Augmented Adaptive Synergies: The Pisa/IIT SoftHand 2. IEEE Transactions on Robotics 2018, 34, 1141 1156.

[18] Tavakoli, M.; Almeida, A. T. D.. Adaptive under-actuated anthropomorphic hand: ISR-SoftHand. In Proceedings of 2014 IEEE/RSJ International Conference on Intelligent Robots and Systems, 14-18 Sept. 2014; pp. 1629-1634.

[19] Rus, D.; Tolley, M. T.. Design, fabrication and control of soft robots. Nature, 2015, 521: 467.

[20] Wang, L.; Iida, F.. Deformation in Soft-Matter Robotics: A Categorization and Quantitative Characterization. IEEE Robotics \& Automation Magazine, 2015, 22: 125-139.

[21] Elango, N.; Faudzi, A. A. M.. A review article: investigations on soft materials for soft robot manipulations. The International Journal of Advanced Manufacturing Technology, 2015, 80: 1027-1037.

[22] Deimel, R.; Brock, O.. A novel type of compliant and underactuated robotic hand for dexterous grasping. International Journal of Robotics Research, 2016, 35: 161-185.

[23] Wei, Y.; Ma, Y.; Zhang, W.. A multi-jointed underactuated robot hand with fluid-driven stretchable tubes. Robotics and Biomimetics, 2018, 5: 2.

[24] Fu, H.; Zhang, W.. The Development of a Soft Robot Hand with Pin-Array Structure. Applied Sciences, 2019, 9: 1011.

[25] Zhou, J.; Yi, J.; Chen, X.; Liu, Z.; Wang, Z.. BCL-13: A 13-DOF Soft Robotic Hand for Dexterous Grasping and In-Hand Manipulation. IEEE Robotics and Automation Letters, 2018, 3: 3379-3386.

[26] Farrow, N.; Li, Y.; Correll, N.. Morphological and Embedded Computation in a Self-contained Soft Robotic Hand. arXiv 2016, arXiv:1605.00354.

[27] Zheng, E.; Zhang, W.. An Underactuated PASA Finger Capable of Perfectly Linear Motion With Compensatory Displacement. Journal of Mechanisms and Robotics. 2019, 11(1):014505..

[28] Sintov, A.; Morgan, A. S.; Kimmel, A.; Dollar, A. M.; Bekris, K. E.; Boularias, A.. Learning a State Transition Model of an Underactuated Adaptive Hand. IEEE Robotics and Automation Letters. 2019, 4(2): 1287-94. 
[29] Sundaralingam, B.; Hermans, T.. Relaxed-rigidity constraints: kinematic trajectory optimization and collision avoidance for in-grasp manipulation. Autonomous Robots. 2019, 43(2): 469-83.

[30] Hota, R. K.; Kumar, C. S.. Effect of hand design and object size on the workspace of three-fingered hands. Mechanism and Machine Theory. 2019, 133: 311-28.

[31] Ellery, A.. Tutorial Review on Space Manipulators for Space Debris Mitigation. Robotics. 2019, 8(2): 34.

[32] Liu, X.; Zhang, X.; Malekian, R.; Sarkodie-Gyan, T.; Li, Z.. Improved Neural Network Control Approach for a Humanoid Arm. Journal of Dynamic Systems, Measurement, and Control. 2019.

[33] Qiao, S.; Guo, H.; Liu, R.; Deng, Z.. Self-adaptive grasp process and equilibrium configuration analysis of a 3-DOF UACT robotic finger. Mechanism and Machine Theory. 2019, 133: 250-66.

[34] Hou, T.; Yang, X.; Aiyama, Y.; Liu, K.; Wang, Z.; Wang, T.; Liang, J.; Fan, Y.. Design and experiment of a universal two-fingered hand with soft fingertips based on jamming effect. Mechanism and Machine Theory. 2019,133: 706-19.

[35] Kawasaki, H.; Mouri, T.. Humanoid Robot Hand and its Applied Research. Journal of Robotics and Mechatronics. 2019, 31(1): 16-26.

[36] Honarpardaz, M.; Ölvander, J.; Tarkian, M.. Fast finger design automation for industrial robots. Robotics and Autonomous Systems. 2019, 113: 120-31.

[37] Lee, Y.; Kim M.; Lee Y.; Kwon J.; Park Y. L.; Lee, D.. Wearable Finger Tracking and Cutaneous Haptic Interface with Soft Sensors for Multi-Fingered Virtual Manipulation. IEEE/ASME Transactions on Mechatronics. 2019, 24(1): 67-77.

[38] Xu, K.; Liu, Z.; Zhao, B.; Liu, H.; Zhu, X.. Composed continuum mechanism for compliant mechanical postural synergy: An anthropomorphic hand design example. Mechanism and Machine Theory. 2019,132: 108-22.

[39] Ostrowska, K.; Gąska, A.; Kupiec, R.; Gromczak, K.; Wojakowski, P.; Sładek, J.. Comparison of accuracy of virtual articulated arm coordinate measuring machine based on different metrological models. Measurement. 2019, 133: 262-70.

[40] Li, Z.; Wu, L.; Ren, H.; Yu, H.. Kinematic comparison of surgical tendon-driven manipulators and concentric tube manipulators. Mechanism and Machine Theory.2017,107: 148-165. 